\title{
Transapical aortic valve implantation after previous mitral valve surgery
}

\author{
Thorsten Drews, MD, Miralem Pasic, MD, PhD, Semih Buz, MD, Axel Unbehaun, MD, \\ Stephan Dreysse, MD, Marian Kukucka, MD, Alexander Mladenow, MD, and Roland Hetzer, MD, PhD
}

\begin{abstract}
Objective: Transcatheter aortic valve implantation is a new method for treatment of very high-risk patients with aortic valve stenosis. Especially in patients who have had previous cardiac surgery, the operative risk can be reduced. Nevertheless, this new procedure has some potential risks in patients with previous mitral valve surgery, owing to the increased risk by direct contact between the 2 valves with inhibition of mechanical mitral leaflet mobility, in view of potential mitral annulus pressure or leaflet damage caused by transcatheter wires.
\end{abstract}

\begin{abstract}
Methods: Between April 2008 and April 2010, 217 consecutive patients (mean EuroSCORE: 40\% \pm 20\%; mean Society of Thoracic Surgeons mortality score: $21 \pm 17 \%$; mean age: $79 \pm 9$ years, 67 men, 150 women) underwent transapical aortic valve implantation. In 6 patients a previous mitral operation ( 1 valve reconstruction, 5 valve replacements) had been performed.
\end{abstract}

Results: In all 6 patients the aortic valve could be implanted by the transapical approach, and the procedure was successful. In 2 patients the heart-lung machine was used electively owing to low ejection fraction $(10 \%$ and $15 \%$ ). In 2 patients there was slight paravalvular leakage of the aortic prosthesis. Neither increased mitral valve regurgitation nor mitral leaflet damage was observed. One patient had endocarditis develop 8 months postoperatively and another with severe reduced left ventricular function died early postoperatively.

Conclusions: Transcatheter valve implantation can be performed successfully after previous mitral valve surgery. Particular care should be taken to achieve optimal valve positioning and not to damage mitral leaflets during manipulation with guide wires. (J Thorac Cardiovasc Surg 2011;142:84-8)

Transapical aortic valve implantation is a new method that might reduce the risk of conventional surgical aortic valve replacement in very high-risk patients. ${ }^{1,2}$ Especially in patients with previous cardiac surgery, the operative risk can be reduced inasmuch as there is less surgical trauma. Nevertheless, previous mitral valve replacement is classified as a contraindication for implantation of the Edwards SAPIEN valve by the manufacturing company (Edwards Lifesciences, Irvine, Calif). Some potential risks exist in patients with previous mitral valve surgery owing to increased risk of endocarditis, potential mitral annulus pressure caused by aortic valve stent dilatation, increased risk of paravalvular leakage owing to asymmetric stent dilatation, the possibility of Edwards SAPIEN valve dislocation owing to poor stent anchorage, direct contact between the 2 valves with inhibition of mechanical mitral leaflet mobility, or biological leaflet damage caused by transcatheter wires.

From Deutsches Herzzentrum Berlin, Berlin, Germany.

Disclosures: Professir Pasic, Dr Drews, Dr Buz, Dr Unbehaun, and Dr Dreysse have been proctors to Edwards Lifesciences since July 2009. There are no other disclosures.

Received for publication Feb 5, 2010; revisions received June 29, 2010; accepted for publication Aug 2, 2010; available ahead of print Sept 30, 2010.

Address for reprints: Miralem Pasic, MD, Phd, Deutsches Herzzentrum Berlin, Augustenburger Platz 1, 13353 Berlin, (E-mail: pasic@dhzb.de).

0022-5223/\$36.00

Copyright (c) 2011 by The American Association for Thoracic Surgery doi:10.1016/j.jtcvs.2010.08.034
In this study, we examine the surgical procedure and the postoperative course of patients undergoing transcatheter aortic valve implantation after previous mitral valve surgery and discuss the potential risks.

\section{METHODS}

Transapical aortic valve implantation was performed between April 2008 and April 2010 in 217 patients at the Deutsches Herzzentrum Berlin. In 41 patients the procedure was performed as a reoperation: in 32 after previous coronary artery bypass grafting, in 12 after previous aortic valve replacement, and in 6 patients after mitral valve surgery. Of these 6 patients, 1 had had mitral valve reconstruction (with Carpentier ring implantation), and 5 patients had had previous mitral valve replacement: St Jude Medical 29 mm (St Jude Medical, Inc, St Paul, Minn), Hancock 33 mm, $29 \mathrm{~mm}$, and $31 \mathrm{~mm}$ (Medtronic, Inc, Minneapolis, Minn), and BjörkShiley $29 \mathrm{~mm}$ (Shiley, Inc, Irvine, Calif) (Table 1).

In all 6 patients, owing to their increased risk for conventional surgery, transapical aortic valve implantation was done using a biological Edwards SAPIEN valve. The procedures were performed in a hybrid operating room in a completely sterile environment and under fluoroscopic imaging with a monoplane angiography system. The surgical technique of transcatheter valve implantation was based on the procedure described by Walther and associates $^{1,2}$ with the modification of transcatheter valve positioning and liberation under simultaneous angiography with contrast medium, not only to make a blind technique visible, but also to find optimal position and to reduce the risk of paravalvular leakage. ${ }^{3}$ Primarily, the intraoperative transesophageal echocardiographic examination was used to control mitral leaflet mobility and ventricular function and to evaluate potential valvular and paravalvular leakage of the Edwards SAPIEN valve. Two patients received 23-mm Edwards SAPIEN valves and the other 4 patients, $26-\mathrm{mm}$ valves. The preoperative and postoperative examinations included clinical and laboratory examinations, electrocardiography, chest 


\section{Abbreviations and Acronyms}

EuroSCORE $=$ European System for Cardiac

Operative Risk Evaluation

SJM $\quad=$ St Jude Medical

STS $\quad=$ Society of Thoracic Surgeons radiography, transthoracic echocardiography, cranial computed tomography, and computed tomography of the chest and pelvis. Preoperative coronary angiography and ultrasound examinations (Doppler) of the arteries and veins of the lower extremities and of the carotid arteries were performed.

\section{RESULTS}

In all 6 patients the technical procedure was performed using the standard technique. ${ }^{1,3}$

\section{Mitral Valve Reconstruction}

One patient had had previous mitral valve reconstruction with ring implantation.

Patient 1. In August 2008, an 82-year-old woman was admitted with severe comorbidities (chronic obstructive lung disease, kidney dysfunction, insulin-dependent diabetes type II, and severe peripheral arteriosclerosis). She had a European System for Cardiac Operative Risk Evaluation (EuroSCORE) of $45 \%$ and a Society of Thoracic Surgeons (STS) mortality score of $23 \%$. A Carpentier-Edwards Physio ring (Edwards Lifesciences) had been implanted for severe mitral valve regurgitation 20 years earlier. The echocardiographic examination showed good left ventricular function, mitral valve regurgitation of grade II, and severe aortic valve stenosis without regurgitation. Owing to severe arteriosclerosis of the peripheral vessels, transapical implantation of a 23-mm Edwards SAPIEN valve was performed. The postoperative echocardiographic check showed good prosthetic function without insufficiency. Eight months later, aortic valve endocarditis developed. The patient required a reoperation, had postoperative pneumonia, and died 4 weeks later of multiorgan failure (Table 1).

\section{Mitral Valve Replacement}

Five patients had had previous mitral valve replacement. Patient 2. We were faced in October 2008 with the case of a 37-year-old woman with severe familial cholesterolemia type II a. She had had a portocaval shunt since 1976, serious calcification of the vessels, and implantation of an apicodescending conduit in May 2004 (owing to severe aortic valve calcification and porcelain aorta). Mitral valve replacement with a mechanical prosthesis (St Jude Medical, $29 \mathrm{~mm}$ ) was performed in February 2004. On admission, the native aortic valve showed severe insufficiency (grade II-III, New York Heart Association classification IV, Euro-
SCORE $85 \%$, STS mortality score $75 \%$ ), leading to global left ventricular failure (ejection fraction 10\%). The situation was first evaluated by our staff and then discussed with the patient's family. Conventional aortic valve replacement was abandoned owing to porcelain aorta, and the decision was made in favor of transapical implantation of a 23-mm Edwards SAPIEN valve through the apicodescending conduit as a last resort. The procedure was performed using the heart-lung machine with femoro-femoral cannulation. Postoperative echocardiographic examination confirmed correct aortic valve function (paravalvular valve insufficiency grade I) and good mitral prosthesis function, but severe left ventricular failure remained (ejection fraction $10 \%$ ). Owing to the still affected left side of the heart, an extracorporeal membrane oxygenator was connected. Unfortunately, the heart function did not recover and the patient died 5 days later of septicemia (Table 1).

Patient 3. In January 2009 we accepted a 75-year-old man with a history of endocarditis. He had had aortic homograft implantation 13 years earlier and mitral valve replacement with a Hancock $33-\mathrm{mm}$ biological valve 2 years earlier. Echocardiographic examination showed no signs of endocarditis; nevertheless, homograft degeneration with insufficiency of grade II-III was seen. There was no impairment of mitral prosthesis function, but severe left ventricular failure (ejection fraction $15 \%$ ). The patient had multiple comorbidities (pulmonary hypertension, atrial fibrillation, kidney failure, hyperlipoproteinemia, and hypertension). The EuroSCORE was $89 \%$ and the STS mortality score $42 \%$. Owing to severe peripheral arteriosclerosis, transapical implantation of a $26-\mathrm{mm}$ Edwards SAPIEN valve was performed. Because of impaired left ventricular function, the heart-lung machine with femoro-femoral cannulation was used. The procedure was technically uncomplicated. Echocardiographic check showed good aortic valve function without insufficiency and an unhindered mitral prosthesis. The patient was extubated on the following day and was discharged 22 days later. Today, 1 year later, the patient has recovered ventricular and good aortic and mitral valve function (Table 1).

Patient 4. The fourth patient with a history of mitral valve replacement was treated in November 2008. The 80-yearold woman with multiple morbidities had a EuroSCORE of $41 \%$ and an STS mortality score of $36 \%$. In 1987 the mitral valve was replaced by a Hancock 29 -mm prosthesis and, owing to valve degeneration, a second valve replacement $(31 \mathrm{~mm})$ was performed in 1997 (Figure 1). On this admission, she had severe aortic valve stenosis with slight regurgitation (grade I). The mitral prosthesis was not impaired. The left ventricular function was slightly reduced (ejection fraction $35 \%$, left ventricular end-diastolic diameter 56 $\mathrm{mm})$. Inasmuch as the iliac artery was small and showed local calcification, transapical implantation of an Edwards SAPIEN valve $(26 \mathrm{~mm})$ was performed and a coronary stent 
TABLE 1. Patients with previous mitral valve surgery

\begin{tabular}{|c|c|c|c|c|c|c|}
\hline Number of patient & 1 & 2 & 3 & 4 & 5 & 6 \\
\hline Sex (male/female) & Female & Female & Male & Female & Female & Female \\
\hline Age (y) & 82 & 37 & 75 & 80 & 82 & 85 \\
\hline Weight $(\mathrm{kg})$ & 61 & 67 & 58 & 56 & 72 & 59 \\
\hline NYHA class* & III & IV & III & III & III & IV \\
\hline FEV (L/100 mL) & $1.03 / 62$ & —* & $1.4 / 53$ & $1.19 / 77$ & $1.19 / 59$ & $1.49 / 68$ \\
\hline EuroSCORE $(\%)$ & 45 & 85 & 89 & 41 & 65 & 45 \\
\hline STS mortality score $(\%)$ & 23 & 75 & 42 & 36 & 50 & 32 \\
\hline $\begin{array}{l}\text { Previous mitral valve } \\
\text { reconstruction/replacement }\end{array}$ & CE Physio ring & SJM (29 mm) & Hancock (33 mm) & Hancock (31 mm) & Hancock (31 mm) & BS $(29 \mathrm{~mm})$ \\
\hline \multicolumn{7}{|l|}{ Echocardiographic data } \\
\hline LV end-diastolic diameter (mm) & 57 & 59 & 60 & 56 & 41 & 45 \\
\hline LV ejection fraction $(\%)$ & 60 & 10 & 15 & 35 & 50 & 50 \\
\hline \multicolumn{7}{|l|}{ Mitral valve } \\
\hline Mitral valve insufficiency (grade) & II & $<\mathrm{I}$ & $<\mathrm{I}$ & I & 0 & $<\mathrm{I}$ \\
\hline \multicolumn{7}{|l|}{ Aortic valve } \\
\hline Aortic valve insufficiency (grade) & 0 & II-III & II-III & I & 0 & I \\
\hline Aortic valve gradient (max/mean $[\mathrm{mm} \mathrm{Hg}])$ & $100 / 70$ & $50 / 40$ & $20 / 10$ & $96 / 60$ & $60 / 50$ & $80 / 54$ \\
\hline Aortic valve orifice area $\left(\mathrm{cm}^{2}\right)$ & 0.45 & 0.7 & 0.8 & 0.6 & 0.7 & 0.6 \\
\hline Diameter of aortic annulus (mm) & 22 & 18 & 22 & 21 & 23 & 20.5 \\
\hline
\end{tabular}

NYHA, New York Heart Association; FEV, forced expiratory volume; EuroSCORE, European System for Cardiac Operative Risk Evaluation; STS, Society of Thoracic Surgeons; $L V$, left ventricular; $C E$, Carpentier-Edwards; SJM, St Jude Medical; $B S$, Björk-Shiley. *Measurement not possible owing to cardiogenic shock.

was implanted in view of coronary artery sclerosis. The operation was performed without the heart-lung machine. The patient was extubated 17 hours later and the postoperative course was uneventful. Echocardiographic examination showed correct prosthetic function without insufficiency. She was discharged on day 20 , and 14 months later she still has good aortic and mitral valve prosthesis function (Table 1).

Patient 5. The fifth patient, an 82-year-old woman, had had an urgent coronary artery bypass graft operation and biological mitral valve replacement using a 31-mm Hancock II prosthesis 4 years earlier. In January 2010 she had severe aortic valve stenosis and severe symmetric calcification. The EuroSCORE was $65 \%$ and the STS mortality score $50 \%$. An Edwards SAPIEN valve $(23 \mathrm{~mm})$ was implanted transapically without the heart-lung machine. She was extubated 1 hour after the operation. The postoperative echocardiographic examination showed correct mitral and aortic valve function; the Edwards SAPIEN valve had slight paravalvular regurgitation (less than grade I). The following course was uneventful (Table 1).

Patient 6. The last patient (female, 85 years old) had had a mechanical mitral valve replacement (Björk-Shiley) 16 years earlier (Figure 2). She was admitted in March 2010 with dyspnea (New York Heart Association classification IV, EuroSCORE $45 \%$, STS mortality score $32 \%),{ }^{4}$ and transthoracic and transesophageal echocardiography confirmed the diagnosis of a severe aortic valve stenosis combined with small valve regurgitation (grade I). The ejection fraction was good $(50 \%)$, and the mitral valve showed good function without stenosis or significant insufficiency (<grade I). The diameter of the aortic valve annulus was $20.5 \mathrm{~mm}$. Owing to symmetric calcification of the 3 aortic valve leaflets, a biological Edwards SAPIEN valve $(23 \mathrm{~mm})$ could be implanted. The iliac arteries were too small for transfemoral access, and a transapical technique was chosen. During introduction of the 26F transapical valve delivery system (Edwards) into the outflow tract of the left ventricle to avoid touching the mitral prosthesis with the crimped valve, the leaflet of the mitral prosthesis was touched by the delivery system, inhibiting its mobility. This made immediate retraction of the delivery system necessary. The remainder of the procedure was uneventful and the patient was extubated 6 hours after the operation. The postoperative echocardiographic examination showed good prosthetic function with minimal central leakage (<grade I) and still good mitral valve function. The patient left the hospital on the 27th postoperative day. Today, 2 months after the operation, she is well, with good aortic and mitral valve function (Table 1).

\section{DISCUSSION}

In patients with previous sternotomy, the operative risk is elevated during conventional aortic valve replacement. The mortality ranged from $3 \%$ to $26 \%{ }^{5,6}$ Although in newer publications a lower mortality risk has been described, ${ }^{7}$ this problem still exists, especially in patients with advanced age and increased STS and EuroSCORE.

Transcatheter aortic valve implantation has been introduced to reduce the surgical risk. Nevertheless, previous 


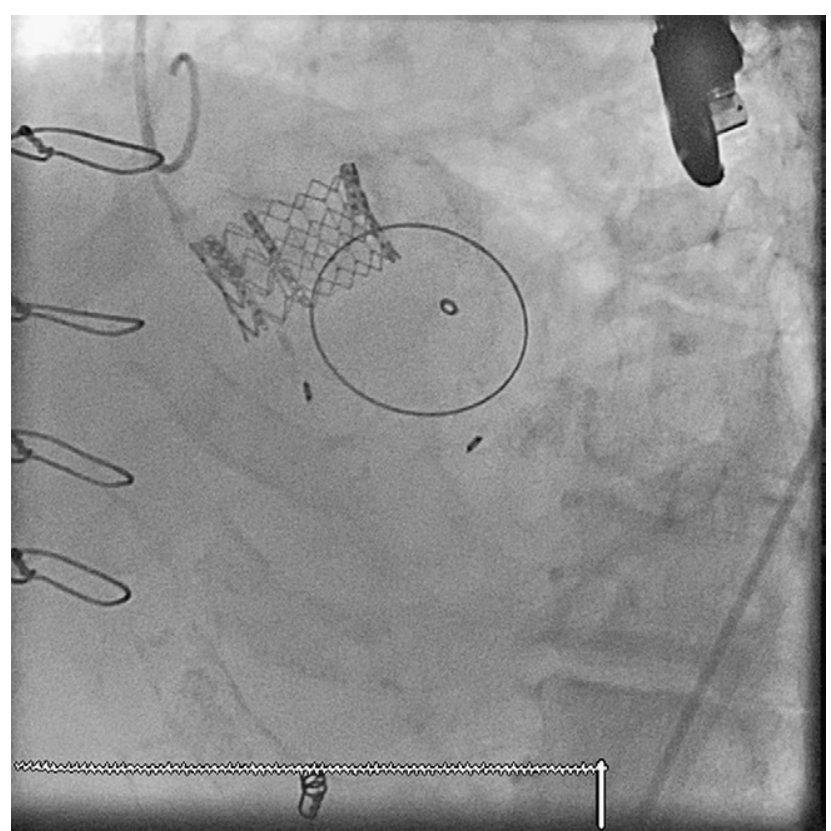

FIGURE 1. Fluoroscopy after transapical valve implantation with Hancock valve $(31 \mathrm{~mm})$ in the mitral valve position (patient 4$)$.

mitral valve replacement is classified as a contraindication for Edwards SAPIEN valve implantation by the manufacturer. This can be explained by several factors: the increased risk of endocarditis, the potential mitral annulus pressure caused by aortic valve stent dilatation, increased risk of paravalvular leakage owing to asymmetric stent dilatation, the possibility of Edwards SAPIEN valve dislocation owing to unsatisfactory stent anchorage, the potential direct contact between the 2 valves with inhibition of mechanical mitral leaflet mobility, and the danger of biological leaflet damage being caused by transcatheter wires. The experience using the transcatheter technique for aortic valve implantation in patients with previous mitral valve surgery is still limited. Recently, a report of retrograde implantation of CoreValve aortic prostheses (CoreValve Inc, Irvine, Calif) in 4 patients with previous mitral valve replacement was published. ${ }^{8}$ Additionally, a case report on an 84-year-old woman with previous mechanical bileaflet mitral valve replacement showed the surgical feasibility of transapical implantation of the Edwards SAPIEN valve. ${ }^{9}$ In this report, the authors recommended higher positioning of the aortic prosthesis.

Our experience consists of 6 patients with different techniques of previous mitral valve surgery. We show that transcatheter wires should be introduced carefully so as not to touch the mitral prosthesis. The 26F transapical valve delivery system (Edwards) should not be introduced into the outflow tract of the left ventricle to avoid touching the mitral prosthesis. Additionally, transcatheter valve positioning and liberation should be done in all cases under

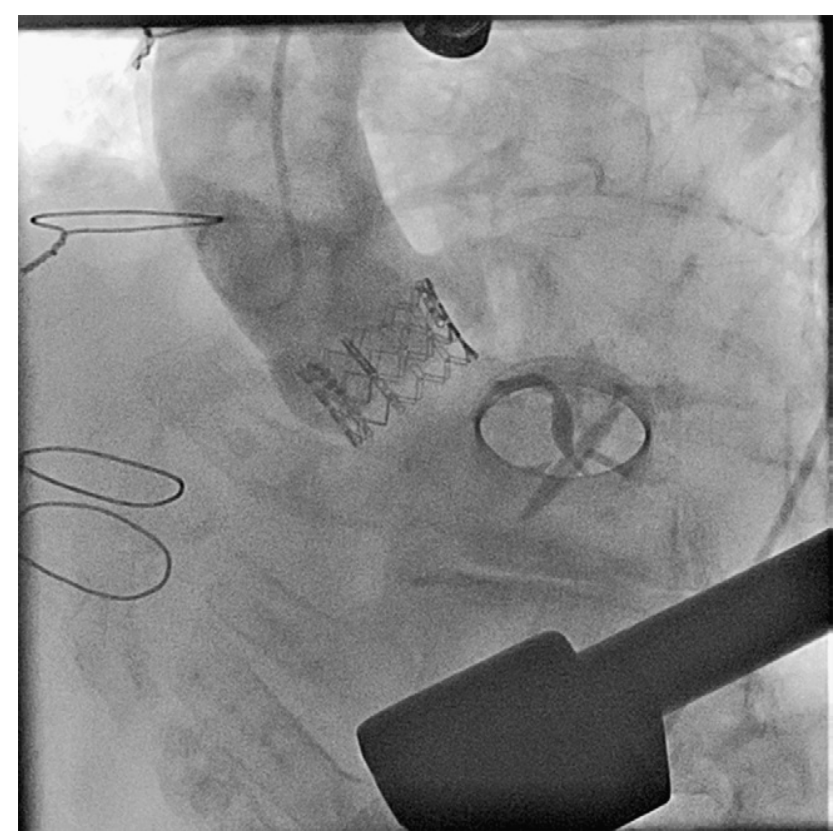

FIGURE 2. Fluoroscopy after transapical valve implantation with BjörkShiley valve $(29 \mathrm{~mm})$ in the mitral valve position (patient 6 ).

simultaneous angiography with contrast medium, not only to make a blind technique visible, but also to find optimal position and to reduce the risk of paravalvular leakage $^{3}$ and contact with the mitral prosthesis. Valve dislocation or increased paravalvular leakage was not observed.

In 1 patient with previous mitral valve reconstruction using a ring implantation, endocarditis occurred postoperatively, making reoperation necessary. The rate of endocarditis in patients with combined aortic and mitral valve surgery is still elevated, making effective prophylaxis for endocarditis necessary. ${ }^{10}$

\section{CONCLUSIONS}

Previous sternotomy is still an increased risk for secondary valve surgery. Transapical aortic valve implantation in patients with previous heart surgery has been in practical use for 2 years, so that this report reflects the early experience. Nevertheless, high-risk patients with previous mitral valve surgery needing aortic valve replacement seem to be optimal candidates for transcatheter valve implantation. With careful introduction of the transcatheter wires under fluoroscopic and echocardiographic control and liberation of the Edwards SAPIEN valve using the "Berlin addition,"3 transapical valve implantation can be safely performed after previous mitral valve surgery, and this should no longer be regarded as a contraindication.

We thank Ms Anne Gale, Editor in the Life Sciences, for editorial assistance. 


\section{References}

1. Walther T, Simon P, Dewey T, Wimmer-Greinecker G, Falk V, Kasimir MT, et al. Transapical minimally invasive aortic valve implantation: multicenter experience. Circulation. 2007;116:I240-5.

2. Walther T, Dewey T, Borger MA, Kempfert J, Linke A, Becht R, et al. Transapical aortic valve implantation: step by step. Ann Thorac Surg. 2009;87:276-83.

3. Pasic M, Dreysse S, Drews T, Buz S, Unbehaun A, Kukucka M, et al. Improved technique of transapical aortic valve implantation: "The Berlin addition" Ann Thorac Surg. 2010;89:2058-60.

4. Roques F, Nashef SA, Michel P, Gauducheau E, de Vincentiis C, Baudet E, et al. Risk factors and outcome in European cardiac surgery: analysis of the EuroSCORE multinational database of 19030 patients. Eur J Cardiothorac Surg. 1999; 15:816-22.

5. Jones JM, O'Kane H, Gladstone DJ, Sarsam MA, Campalani G, MacGowan SW, et al. Repeat heart valve surgery: risk factors for operative mortality. $J$ Thorac Cardiovasc Surg. 2001;122:913-8
6. Biglioli P, Di Matteo S, Parolari A, Antona C, Arena V, Sala A. Reoperative cardiac valve surgery: a multivariable analysis of risk factors. Cardiovasc Surg. 1994;2:216-22.

7. LaPar DJ, Yang Z, Stukenborg GJ, Peeler BB, Kern JA, Kron IL, et al. Outcomes of reoperative aortic valve replacement after previous sternotomy. J Thorac Cardiovasc Surg. 2010;139:263-72.

8. Bruschi G, De Marco F, Oreglia J, Colombo P, Fratto P, Lullo F, et al. Percutaneous implantation of CoreValve aortic prostheses in patients with a mechanical mitral valve. Ann Thorac Surg. 2009;88:e50-2.

9. Scherner M, Strauch JT, Haldenwang PL, Baer F, Wahlers T. Successful transapical aortic valve replacement in a patient with a previous mechanical mitral valve replacement. Ann Thorac Surg. 2009;88:1662-3.

10. Panda BR, Shankar R, Kuruvilla KT, Philip MA, Thankachen R, Shukla V, et al. Combined mitral and aortic valve replacement for rheumatic heart disease: fifteen-year follow up and long-term results. J Heart Valve Dis. 2009; 18:170-9. 